\section{Marlene Haffner}

As head of the Office of Orphan Products Development, Marlene Haffner offers a perspective on the immense influence the Orphan Drug Act has had in promoting research on and awareness of rare diseases.

For the vast majority of us, the labels on food packaging warning 'this product contains phenylalanine' are a curiosity, but for individuals diagnosed with phenylketonuria (PKU), they serve as an important aid in managing their disease. Increased awareness of this debilitating rare disease, which can cause brain damage if not managed properly, led to this increased labeling of consumer products. However, people suffering from PKU have problems not only in knowing what they shouldn't eat, but also in feeling normal while they follow their approved diet. This problem inspired scientists to create 'normal-looking' food for those with PKU, thus helping them stick to the correct regimen. The organization that helped make this research possible is the Office of Orphan Product Development (OOPD), which has been charged with the task of administering the Orphan Drug Act for the last 24 years. Marlene Haffner, the director of this office for the last 19 of those years, is pleased with the current success in orphan drug development but notes that there is still much to do.

The Orphan Drug Act was inspired by the recognition that the development of treatments for rare diseases, although an imperative in the medical community, was not likely to be a profitable task. Though a cost analysis is probably far from the minds of academicians, who may pursue a topic more out of interest or curiosity than with the express purpose of treating a disease, it is of relevance to scientists at companies, for whom profit has to be an important consideration. The act thus provides grants toward and market exclusivity for any drug that treats a rare disease (an 'orphan' drug), thereby reducing or justifying the costs of researching and testing the product. Indeed, Haffner says "I think the law was written in a way that encouraged the pharmaceutical industry to become involved in these products, and it worked." She notes that start-up biotech companies have been particularly likely to pursue orphan drugs, as the profits shown by these drugs are more in line with the expectations of such companies than with those of a large pharmaceutical firm.

Rare diseases, according to the Orphan Drug Act, are generally defined as those affecting less than 200,000 people in the United States, but this number may be higher in cases where the costs associated with developing a drug are not anticipated to be recovered. In practice, however, rare diseases may affect only a handful of people; indeed, one of the early successes made possible by Haffner's office was the drug PEG-ADA, which was initially used to treat about 12 people with adenosine deaminase (ADA) deficiency. Haffner notes that it is of critical importance to raise awareness of the existence of both the disease and the treatment because "when one has a good treatment, more patients seem to be diagnosed." For example, in contrast to the 125,000 people reported to have multiple sclerosis back when the OOPD certified it as a rare disease, Haffner notes, "the first interferon to treat MS was approved, and within two weeks, more than 200,000 people had signed up to receive the drug."

The OOPD serves several functions, ranging from awarding grants to certifying patient populations, and is working with scientists to develop drugs, devices and foods for use in small populations. The administration of the Orphan Drug Act has proved extremely successful; indeed, the number of approved drugs to treat rare diseases has grown from 10 prior to the passage of the law to approximately 300 today (with another $\sim 1,350$ candidates designated by the office and under investigation). In addition to spurring on new research, Haffner says "products that had been floating around for a long time could now be fully developed because there was a way to protect them that had not previously existed." For example, the human immunodeficiency virus was an early 'rare disease', as less than 10,000 patients existed when the virus was identified and the first people diagnosed. Haffner recalls, "no one could have predicted that HIV would in time levy such a toll on the world." As a result, she notes, "AZT, the first product to treat HIV, was an orphan product." In fact, Haffner adds, “AZT was a product that wasn't thought to be worthwhile developing because the patent had expired. Without the Orphan Drug Act, it might have been quite some time before that product came to market."

The success of the Orphan Drug Act here in the United States is having repercussions around the globe. "People in other countries, seeing

"No one could have
predicted that the human
immunodeficiency virus
would in time levy such a toll
on the world. AZT, the first
product to treat HIV, was an
orphan product."

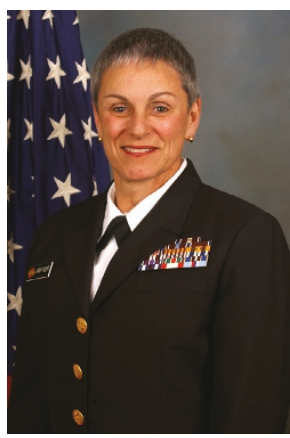

the success of the US Orphan Drug Act, began to lobby their respective governments, with the result that there are now many orphan drug programs throughout the world." In thinking about how the international community can work together to forward the progress of medicine, the "FDA has already signed, with the European Union, a memorandum of understanding having to do with parallel development of products." The agreements put in place in this memorandum will likely pave the way for increased cooperation in developing orphan drugs as well. Improved international interactions would help companies find orphan products profitable as their patient numbers increase, but will also require careful attention in defining rare diseases on a global scale.

Within the United States, Haffner cites the all-too-familiar problem of lack of resources as a major hurdle. Haffner says, "I'd love to have the ability to be more proactive towards finding and encouraging products to treat rare diseases, because we just are spread too thin to be able to follow up on some things." This problem extends to the inability not only to be in contact with ongoing projects, but also to support new projects. She laments, "There are many, many excellent grants that go unfunded because we don't have enough money to fund them all." Yet Haffner is committed to the long-term goal, saying, "I'm a problem solver. I like to find ways to get things done."

In thinking about the impact of the OOPD, Haffner muses, "Certainly the office has brought more awareness to rare diseases, to drugs for rare diseases, and the ability to develop products for rare diseases while still being profitable." In an economy where most everyone is looking for the next big thing, her office helps to ensure that at least a few people are looking for the next small thing.

Catherine Goodman, Boston, Massachusetts 